\title{
Benefit of interleaved practice of motor skills is associated with changes in functional brain network topology that differ between younger and older adults
}

Chien-Ho (Janice) Lin ${ }^{\mathrm{a}, \mathrm{b}}$, Barbara J. Knowlton ${ }^{\mathrm{c}}$, Allan D. Wu ${ }^{\mathrm{d}, \mathrm{e}}$, Marco Iacoboni ${ }^{\mathrm{e}, \mathrm{f}}$, Ho-Ching Yang ${ }^{\mathrm{g}}$, Yu-Ling Ye ${ }^{\mathrm{g}, \mathrm{h}}$, Kuan-Hong Liu ${ }^{\mathrm{g}}$, and Ming-Chang Chiang ${ }^{\mathrm{g}}$

${ }^{a}$ Department of Physical Therapy and Assistive Technology, National Yang-Ming University, Taipei, 112, Taiwan

${ }^{\text {b} Y e o n g-A n ~ O r t h o p e d i c ~ a n d ~ P h y s i c a l ~ T h e r a p y ~ C l i n i c, ~ T a i p e i, ~ 112, ~ T a i w a n ~}$

'Department of Psychology, University of California, Los Angeles, California 90095, USA

${ }^{\mathrm{d}}$ Department of Neurology, David Geffen School of Medicine, University of California, Los Angeles, California 90095, USA

eAhmanson-Lovelace Brain Mapping Center, University of California, Los Angeles, California 90095, USA

${ }^{\mathrm{f}}$ Department of Psychiatry and Biobehavioral Sciences, University of California, Los Angeles, California 90095, USA

${ }^{g}$ Department of Biomedical Engineering, National Yang-Ming University, Taipei, 112, Taiwan

${ }^{\mathrm{h}}$ Department of Diagnostic Radiology, Chang Gung Memorial Hospital, Chiayi, 613, Taiwan

Submitted to Neurobiology of Aging: July 1, 2015

Revised Version submitted: December 11, 2015

Abbreviated title: Age affects brain networks for motor learning

\section{Abstract: 170 words; page count: 33 pages}

Figures: 5, Table: 0

Email: Chien-Ho Lin (chienho.lin@gmail.com), Barbara J. Knowlton (knowlton@psych.ucla.edu), Allan D.Wu (allanwu@mednet.ucla.edu), Marco Iacoboni (iacoboni@ucla.edu), Ho-Ching Yang (kawc1034@gmail.com), Yu-Ling Ye (daydream_1014@hotmail.com), Kuan-Hong Liu (mai_k319@hotmail.com), Ming-Chang Chiang (mcchiang@ym.edu.tw)

Address Correspondence: Ming-Chang Chiang, Department of Biomedical Engineering, National Yang-Ming University, No.155, Sec.2, Linong Street, Taipei, 112, Taiwan, Phone 886-2-2826-7110, Fax 886-2-2821-0847, Email: mcchiang@ym.edu.tw 


\section{ABSTRACT (170 words; 170 words max.)}

Practicing tasks arranged in an interleaved manner generally leads to superior retention compared to practicing tasks repetitively, a phenomenon known as the contextual interference (CI) effect. We investigated the brain network of motor learning under CI, i.e., the CI network, and how it was affected by aging. 16 younger and 16 older adults practiced motor sequences arranged in a repetitive or an interleaved order over 2 days, followed by a retention test on Day 5 to evaluate learning. Network analysis was applied to functional MRI data on retention to define the CI network by identifying brain regions with greater between-region connectivity following interleaved compared to repetitive practice. CI effects were present in both groups but stronger in younger adults. Moreover, CI networks in younger adults exhibited efficient small-world topology, with a significant association between higher network centrality and better learning following interleaved practice. Older adults did not show such favorable network properties. Our findings suggest that aging affects the efficiency of brain networks underlying enhanced motor learning following CI practice.

Keywords: contextual interference, serial reaction time task, psychophysiological interaction, functional connectivity, small world networks, functional magnetic resonance imaging 


\section{INTRODUCTION}

Challenging learners by increasing difficulty during skill practice may enhance their long-term retention performance, a strategy known as desirable difficulty (Christina and Bjork, 1991). One way to increase desirable difficulty in motor practice is to increase its contextual interference (CI). Practicing tasks in an interleaved order (higher CI) generally results in inferior practice performance but superior retention compared to practicing in a repetitive order (lower CI) (Shea and Morgan, 1979). In our previous work investigating aging effects on motor sequence learning, we found that older adults could benefit from high CI practice as did younger ones; they exhibited superior retention following interleaved compared to repetitive practice (Lin et al., 2010; Lin et al., 2012a). However, functional magnetic resonance imaging (fMRI) showed that the above CI effects on motor learning involved different neural substrates in younger and older adults: (1) the benefits of CI were associated with activations in the rostral prefrontal cortex and the sensorimotor regions in older adults, but in the dorsolateral prefrontal cortex in younger adults (Lin et al., 2012a); (2) the CI benefits in younger and older adults involved different functional connectivity patterns of motor areas. Using psychophysiological interaction (PPI) analysis (Friston et al., 1997), we found that interleaved practice enhanced functional connectivity between the prefrontal and inferior frontal cortices in younger adults, but between bilateral prefrontal and premotor-parietal cortices in older adults (Lin et al., 2012b).

Here we extend our previous work by combining PPI (Gerchen et al., 2014) and network methods (Rubinov and Sporns, 2010) to investigate how aging influences the functional organization of the brain underlying the CI effects on motor sequence learning. We modeled changes in the spatial pattern of whole-brain functional connectivity due to interleaved practice as a network, denoted as the CI network. In this CI network, one brain region, i.e., a node of the network, was connected with another if the higher CI, or interleaved practice condition, led to a stronger temporal association between activities in these two regions during retention than the lower CI, or repetitive condition, as outlined in Figure 1. Compared to previous work that focused on contributions from single brain 
regions, network approaches have opened up a new horizon to understand learning from the wholebrain perspectives (Sami and Miall, 2013). Moreover, with the help of graph theoretical methods, the complex structure of a brain network may be characterized using topological measures that quantify the geometrical properties of the network (Rubinov and Sporns, 2010). One important topological feature found in both biological and non-biological networks is the small-world topology, where the networks are in an optimal balance between functional integration and segregation (Watts and Strogatz, 1998). A small-world network is therefore highly efficient in communication across different regions in the brain (Bassett and Bullmore, 2006), and associated with higher intellectual performance (van den Heuvel et al., 2009). Under pathological conditions, the small-world configuration of brain networks may be disrupted (Supekar et al., 2008). To our knowledge, it remains unclear whether a small-world brain network is associated with enhanced motor learning, and if it is, whether this small-world property is altered by normal aging.

To address the above issues, we compared the CI networks between healthy younger and older adults. We hypothesized that the CI network in younger adults during retention would be more efficient than that in older adults; in other words, the younger-adult network would exhibit a small-world topology. We further hypothesized that enhanced network efficiency would be associated with the benefits of CI to skill learning, so that (1) the magnitude of the CI effect on motor sequence learning would be higher in younger than older adults, as younger adults were expected to have a more efficient brain network configuration, and (2) better communication within the CI network would be associated with better retention after interleaved practice.

\section{METHODS}

\subsection{Participants}


Sixteen younger ( 9 men and 7 women, age $=26.4 \pm 3.1$ years, education $=17.2$ years $)$ and 16 older (7 men and 9 women, age $=66.2 \pm 4.7$ years, education $=16.5$ years; mean \pm SD) right-handed adults gave written informed consent to participate the current study approved by the local Institutional Review Board. The two cohorts were the same as those in our previous paper (Lin et al., 2012a). They were recruited from the University of California, Los Angeles, and the adjacent community. Participants were excluded if they were a musician or a professional typist, had score less than 28 on the Mini-Mental State Exam (MMSE; (Folstein et al., 1975)), any contraindications to MRI, uncorrected vision loss, or significant medical conditions that prevented them from performing the task. The gender distribution and the level of education were not significantly different between the two groups ( $P=0.57$ and $P=0.42$ respectively).

\subsection{Experimental protocols for the serial reaction time (SRT) task during fMRI scanning}

The participants practiced the serial reaction time (SRT) task during fMRI scanning on two consecutive training days (Days 1 and 2), and were tested for their delayed retention performance on Day 5. The experimental design is explained in Fig. 1, as has been reported in our previous publication (Lin et al., 2012a). On each trial of the SRT task the participants were instructed to respond to one of three different 4-element sequences as quickly and as accurately as possible, where each sequence was represented by a specific permutation of 4 colored circles (Fig. 1a and b). Functional images were acquired while the participants performed the SRT task inside a Siemens Trio 3.0 T MRI scanner. We applied a blocked design for fMRI, with 18-second task blocks interleaved with 18-second rest blocks. A custom-designed computer software program written with Presentation (Neurobehavioral Systems, Berkeley, CA) controlled the appearance of the colored circles and recorded the participants’ responses. Response times (RT), defined as the time between stimulus onset and key press, were recorded for each key press. The total RT for every 4-element sequence trial was calculated by adding up the RT of each of the four key presses. During the task blocks, each sequence was presented for a fixed duration of 3 seconds. If the participant completed a sequence before 3 seconds, 4 transparent 
circles would appear on the screen to keep the participants' attention and reduce eye movements.

During the rest blocks, the circles were replaced by a fixation cross in the center of the screen.

There were three fMRI runs respectively on Days 1 and 2. Each run consisted of 54 trials of sequences lasting for a total of 348 seconds, with 153 echo-planar imaging (EPI) volumes acquired (gradient-echo, $\mathrm{TR}=2,000, \mathrm{TE}=30$, flip angle $=90^{\circ}$ ). Each EPI volume had 34 transverse 4-mm slices with 1-mm gap, and a 64 X 64 matrix yielding an in-plane resolution of $3 \mathrm{~mm} \mathrm{X} 3 \mathrm{~mm}$. Each participant was assigned to either the Repetitive practice (RP) or Interleaved practice (IP) condition, where the arrangement of the sequences in these two conditions was described in Fig. 1c. On Day 5, there were also three fMRI runs. In the first two runs the participants were tested with the three sequences they had practiced on Days 1 and 2, presented either in a repetitive order (denoted by Repetitive testing condition) or in an interleaved order (denoted by Interleaved testing condition). The third run consisted of three novel sequences to assess whether learning was specific to the training sequences (Fig. 1c). Each run consisted of 36 trials of sequences lasting for 228 seconds as a total (99 EPI volumes). Compared to 54 trials per run on Days 1 and 2, the duration of the fMRI runs on Day 5 was shortened in order to limit further learning (Cross et al., 2007). We applied a within-participant cross-over design to control for inter-participant variability in learning ability. Each participant underwent either the Repetitive practice (RP) followed by the Interleaved practice (IP) condition at least two weeks later, or vice versa. The order of the practice conditions was counter-balanced across the participants.

We focused the subsequent analyses on the behavioral and fMRI data acquired during the Interleaved testing condition on the retention day (Day 5), as we were interested in how aging modulated the CI effect of practice on retention performance. Moreover, in our previous work we found that the CI effect of practice was significant in the Interleaved but not Repetitive testing condition during retention (Lin et al., 2012a). 


\subsection{Psychophysiological interaction (PPI) analysis}

We used PPI analysis (Gitelman et al., 2003) to identify any pair of brain regions where interleaved practice led to greater connectivity between them than repetitive practice. PPI models how neural activity in one brain region (the seed region) differently influences the activity in another brain region (the target region) when there are changes in a cognitive/perceptual process (e.g. Interleaved versus Repetitive condition) (Fig. 1d). To investigate neural substrates for the CI effect on motor learning in the context of a network, we adopted the multi-regional PPI approach (Gerchen et al., 2014) where PPI was performed across 88 cortical and subcortical regions defined in the Anatomical Automatic Labeling (AAL) atlas (http://www.gin.cnrs.fr/spip.php?article217), with each of the 88 brain regions serving as the seed, and the other 87 regions separately as the target. The name and abbreviation of the AAL atlas regions are listed in Supplementary Table 1. Compared to traditional methods that select seeds from areas of significant fMRI activation (Gitelman et al., 2003), seed selection based on structural anatomical atlases involves less a priori assumptions about the functional parcellation of the brain, provides a more comprehensive coverage of potentially important areas in brain networks, and has been adopted in most functional network studies (Gerchen et al., 2014; Stanley et al., 2013). Nevertheless, the fMRI-based seed-selection methods may complement the multi-regional PPI approach by providing more information about the functional significance of brain networks. As such, as a follow-up analysis, we also performed PPI based on seeds selected from regions where interleaved practice led to stronger functional connectivity than repetitive practice with the premotor cortex (PMC) or dorsolateral prefrontal cortex (DLPFC) during retention (Day 5) (Lin et al., 2012b). See Supplementary Methods for the seed-selection procedures. We selected these PMC- or DLPFCrelated regions as the seed regions because functional connectivity between these regions and PMC or DLPFC was associated with benefits of CI to motor learning (Lin et al., 2012b). These seed regions are listed in Supplementary Table 2. 
Functional images were first pre-processed using the Statistical Parametric Mapping software (SPM8, Wellcome Department of Cognitive Neurology, London, UK), as detailed in the Supplementary Methods. Functional connectivity estimated using PPI was based on a multiple regression model, where the fMRI time-series data of the target region was regressed against: (1) the "physiological variable", which was the fMRI time-series data in the seed region, (2) the "psychological variable”, which was a vector specifying the task condition (1 for Interleaved practice and -1 for Repetitive practice) convolved with the hemodynamic response function (HRF), and (3) the interaction term, which was the product of the psychological variable and the BOLD data deconvolved with the HRF. For each participant on the retention day (Day 5), the PPI analysis yielded an $88 \times 88$ matrix. The element in row $i$ and column $j$ of the matrix was a $t$-contrast derived from the regression coefficient with respect to the PPI interaction term that specified the modulatory effect of the psychological variable on the functional connectivity from the seed (region $i$ ) to the target region (region $j$ ). The above participant-level t-contrast matrices of the younger and older adults were then respectively entered into a random-effects group analysis using the one-sample t-test, yielding a grouplevel connectivity matrix of $t$-values respectively for the younger and older adults that gauged the significance of excess in functional connectivity for the Interleaved over the Repetitive condition (Interleaved minus Repetitive) between the seed and the target region (Fig. 1e).

\subsection{Generation of the CI network}

The above group-level connectivity matrix of the younger or older adults was binarized at a threshold of $t>3$, so that any entry of 1 in the binarized connectivity matrix, say the element in row $i$ and column $j$, indicated a one-way functional link for node $i$ (i.e., the seed region $i$ ) connecting to node $j$ (i.e., the target region $j$ ) with greater strength in the Interleaved condition than the Repetitive condition. Multiple comparisons across the connectivity matrix due to PPI regression for every pair of seed and target regions were corrected using permutation testing implemented in the Network Based 
Statistic (NBS) Toolbox (https://sites.google.com/site/bctnet/comparison/nbs) (Zalesky et al., 2010). A total of 1000 permutations were generated by randomly assigning the "Interleaved minus Repetitive" (i.e., assigning 1 for Interleaved and -1 for Repetitive practice condition in the PPI regression model) or “Repetitive minus Interleaved” (i.e., assigning - 1 for Interleaved and 1 for Repetitive practice in the PPI regression model) contrast to each participant in the group analysis. This was based on the null hypothesis that there was no difference in the connectivity strength between the Interleaved and Repetitive conditions. For each permutation, a new group-level connectivity matrix was recalculated and binarized at the same threshold of $t>3$. The size of each set of connected nodes, denoted as cluster, in the original and permuted connectivity matrices was computed. The family-wise error rate (FWER), or the corrected $P$-value, of the original connectivity matrix was then defined as the proportion of the 1000 permuted matrices with their maximal cluster size greater than that of the original connectivity matrix. Overall significance was achieved if the corrected $P$-value $<0.05$. The original connectivity matrix was thresholded again so that only clusters surviving the size threshold of FWER $<0.05$ were preserved, generating a directed network mediating the CI effect, or the CI network (Fig. 1f). Solitary nodes, i.e., nodes without any connection to the other nodes in the network, were excluded for the subsequent graph theoretical analysis (see below).

\subsection{Graph theoretical analysis}

We applied graph theoretical analysis to the CI network derived using the above PPI method to assess functional interactions across the brain for the CI effect of motor learning. The CI network was characterized by graph theoretical measures including: (1) the betweenness centrality that identified key regions mediating the CI effect on learning, i.e., the hubs of the CI network, (2) the small-world topology that indicated the efficiency of functional integration among brain regions underlying the CI retention effect, and (3) the network modularity index that assessed functional segregation of the brain regions in the CI network (Rubinov and Sporns, 2010). These graph theoretical measures are briefly 
described below, with their mathematical definitions and formulae detailed in the Supplementary Methods.

(1) Betweenness centrality: The betweenness centrality (BC) of a node is defined as the fraction of all shortest paths in the network passing through this node (Rubinov and Sporns, 2010). As such, a node with higher BC indicates that this node serves as a hub in the network, controlling the information flow passing through this node (Valente et al., 2008). Here we defined a node with BC above the $90^{\text {th }}$ percentile of all nodes as the hub node of the CI network (He et al., 2009). Moreover, it has been shown that the mean BC of a network increased with more nodes randomly removed from the network (Testa et al., 2014). As such, a network with higher mean BC enables more efficient communication within the network because the network has less redundant nodes and therefore information exchanges depend more on the hub nodes lying along the geodesic pathways of other nodes in the network (Valente et al., 2008).

(2) Small-world topology: A brain network is in a small-world topology if it is simultaneously highly segregated and integrated, a configuration that allows for efficient information processing (Rubinov and Sporns, 2010). The small-world topology of a network may be quantitatively represented by the small-world index $S$ that measures the balance between local segregation and global integration of the network. A network is regarded to be small-world if $S>1$ (Humphries and Gurney, 2008) . (3) Network modularity index: The modularity index $Q$ assesses whether a network can be divided into non-overlapping components, or modules (Rubinov and Sporns, 2010). A higher $Q$ indicates that connections between the nodes were denser within a module but sparser between different modules, suggesting the network is more functionally segregated. The significance of $Q$ was assessed as a $Z$ score estimated by comparing the value of Q of the real network with that of the 100 degree-matched random networks (Yan and He, 2011). See Supplementary Methods for how such random networks were generated. 
Comparisons of the network properties between the younger and older adults were performed using a two-level permutation procedure. The first-level permutation was to generate 1000 permuted networks respectively for the younger and older adults by randomly exchanging the group (younger versus older adults) to which a participant belonged, followed by the one-sample t-test to calculate the group-level connectivity matrix that was subsequently binarized at a threshold of $t>3$. This was based on the null hypothesis that there was no difference in network properties between the two groups. For each first-level permuted network, we applied a second-level permutation to identify the clusters that survived the threshold of FWER $<0.05$ at the network level. 1000 second-level permuted networks were generated by randomly exchanging the "Interleaved minus Repetitive” and "Repetitive minus Interleaved" contrasts across the participants (see Section 2.4 above). The size threshold of FWER <

0.05 was then defined as the $95^{\text {th }}$ percentile of the distribution for the maximal cluster size of the 1000 second-level permuted networks. The statistical significance of the difference in network properties between the younger and older adults was represented by a $P$-value defined as the proportion of the 1000 first-level permuted networks for which the difference in network properties was greater than the observed difference with respect to the real CI networks between the two groups. A $P<0.05$ indicated statistical significance.

\subsection{Statistical analysis}

To quantify the benefits of interleaved practice to learning, in our previous work we computed the difference in the mean response time (RT) during retention on Day 5 for the Repetitive minus the Interleaved condition, denoted by the R-I contrast. Since the CI effect in sequence learning was defined as how much improvement in retention performance following interleaved compared to repetitive practice, a higher R-I contrast then indicated a stronger CI effect (Lin et al., 2012a). However, simply using the R-I contrast may underestimate the benefits of CI learning to the younger adults because they had a shorter retention RT than the older adults following repetitive practice (see Results) and as such had less room than the older adults to improve their retention performance through interleaved practice, 
as shown in Fig. 2a. To correct for this ceiling effect for the younger adults, we used the analysis of covariance (ANCOVA) method to adjust for the differences in the baseline measures (i.e., retention RT following repetitive practice) between the younger and older adults, where retention RT following repetitive practice was treated as a covariate (Vickers, 2001). ANCOVA provides an unbiased estimation of the true experimental effect in the presence of differences in the baseline measures, and has proven to be statistically more powerful than using the absolute difference or percentage change for the post-experimental measure relative to the baseline one (Vickers, 2001). Here the retention RT following interleaved practice $\left(\mathbf{t}_{\mathbf{I P}}\right)$ was regressed against the participant's group ( $\mathrm{g}$; $\mathrm{g}=1$ for younger and 0 for older adults) as the explanatory factor, and the retention RT following repetitive practice $\left(t_{\mathrm{RP}}\right)$ as the covariate, given by

$$
\mathrm{t}_{\mathrm{IP}}(i)=\beta_{0}+\beta_{\mathrm{g}} \mathrm{g}(i)+\beta_{\mathrm{RP}} \cdot \mathrm{t}_{\mathrm{RP}}(i)+\varepsilon(i)
$$

The symbol $i$ in the parentheses represents participant $i ; \beta_{0}$ and $\varepsilon$ are respectively the constant and residual error of the regression. Under the ANCOVA model, the influences of $\mathbf{t}_{\mathbf{R P}}$ on $\mathbf{t}_{\mathbf{I P}}$ were eliminated so that the CI effect, i.e., the improvement in learning performance following interleaved over repetitive practice, in younger and older adults was compared as if $\mathbf{t}_{\mathbf{R P}}$ in both groups was equal to $\overline{\mathbf{t}}_{\mathbf{R P}}$, where $\overline{\mathbf{t}}_{\mathbf{R P}}$ is the mean value of $\mathbf{t}_{\mathbf{R P}}$ across all the participants (Fig. 2b). By this way, the CI effect on motor learning after the above adjustment was represented by the adjusted $R$-I contrast, given by $\overline{\mathbf{t}}_{\mathrm{RP}}-\hat{\mathbf{t}}_{\mathrm{IP}}(\boldsymbol{i})$, where $\hat{\mathrm{t}}_{\mathrm{IP}}(i)$ is the value of $\mathrm{t}_{\mathrm{IP}}$ for participant $i$ adjusted for group differences in $\mathrm{t}_{\mathrm{RP}}$, expressed as $\hat{\mathrm{t}}_{\mathrm{IP}}(i)=\mathrm{t}_{\mathrm{IP}}(i)-\beta_{\mathrm{RP}} \cdot\left[\mathrm{t}_{\mathrm{RP}}(i)-\overline{\mathrm{t}}_{\mathrm{RP}}\right]$.

We next tested the association between the adjusted R-I contrast and the mean betweenness centrality of the network in each participant using Spearman's rank correlation, to examine the hypothesis that a greater learning benefit following practice with higher CI is associated with better communication within the CI network, i.e., information is more likely to flow through the shortest paths in the network. The CI network for each participant was defined using the "multiple-thresholds" 
approach (Langer et al., 2013; van den Heuvel et al., 2009), where the participant-level $t$-contrast matrix derived from PPI was thresholded at $t>t_{c} . t_{c}$ increased from 3.0 to 4.5 with an increment of 0.1. The $t$-contrast matrix became too sparse to yield a meaningful network when $t_{c}$ was greater than 4.5. Differences between the younger and older adults in the strength of the above associations were tested using a multiple regression model including the mean betweenness centrality of the network as the dependent variable, and Group (1 for younger adults and -1 for older adults), CI (values of the adjusted R-I contrast), and Group $\times$ CI interaction as the independent variables. A significant Group $\times$ CI interaction then indicated that the difference between the younger and older adults in the above associations between the learning benefit and network centrality was significant (Nieuwenhuis et al., 2011). To correct for multiple comparisons across these $16 t_{c}$ 's (for $t_{c}$ ranging from 3.0 to 4.5 ), the association of the learning benefit and network centrality or Group $\times$ CI interaction was regarded significant if the corresponding $P$-value was less than the Bonferroni's threshold equal to 0.003125 (= 0.05/16).

\section{RESULTS}

\subsection{Younger adults exhibited a stronger CI effect on motor sequence learning than older adults}

Both younger and older adults exhibited a shorter RT on retention following practice in the Interleaved than the Repetitive condition, with a positive R-I contrast (R-I contrast $=217.5 \pm 47.9 \mathrm{~ms}$ in the younger adults, and $195.9 \pm 69.7 \mathrm{~ms}$ in the older adults). However, the difference in the R-I contrast between the two groups was not significant $(P=0.8)$ (Fig. 2a). Since the younger adults had a shorter retention RT than older adults following repetitive practice (retention RT following repetitive practice $=1114.9 \pm 74.4 \mathrm{~ms}$ in the younger and $1738.6 \pm 74.2 \mathrm{~ms}$ in the older adults, $P<0.0001$ ), to correct for the ceiling effect in CI learning for the younger adults, the R-I contrast was adjusted for the retention RT following repetitive practice using ANCOVA. The younger adults showed a higher adjusted R-I 
contrast (i.e., the CI effect) on sequence learning than the older adults (adjusted R-I contrast $=387.5 \pm$ $21.2 \mathrm{~ms}$ in the younger adults, and $26.0 \pm 58.6 \mathrm{~ms}$ in the older adults; $P=0.0006$ ) (Fig. $2 \mathbf{b}$ ).

\subsection{The CI networks based on the AAL atlas}

There are two main findings with respect to the CI networks generated based on seed regions selected from the AAL atlas:

(1) The CI network in younger adults but not older adults was in a small-world topology: For the younger adults, we identified a 13-node CI network during retention (Day 5), with $P=0.04$ corrected for multiple comparisons using the NBS method (Fig. 2c, left, and Fig. 3). The completeness of the network was equal to $24.36 \%$, meaning that 38 directed connections were identified out of 156 . The CI network was highly modular, with a modularity index $Q=0.5(Z$-score $=10.65)$ significantly higher than that of random networks. The network could be divided into two separate modules: the left frontotemporal (module 1) and the left occipital (module 2) areas. Nodes in each module formed a smallworld network, with the small-world index $S=1.88$ in the left fronto-temporal module, and $S=6.12$ in the left occipital module. The hubs of the CI network were the inferior frontal gyrus, orbital part (Frontal_Inf_Orb_L; BC $=0.15$ ), and the middle temporal pole $($ Temporal_Pole_Mid_L; BC $=0.15$ ), both in the left hemisphere. Both hubs belonged to the left fronto-temporal module.

For the older adults, we identified a CI network on Day 5 (with a corrected $P$-value $=0.005$ ) (Fig. 2c, right, and Fig. 4). Compared to the CI network of the younger adults on the same day, the CI network in the older adults was larger in size, with 45 nodes (compared to 13 nodes in the younger adults). The completeness of the network was also slightly higher in the older adults, with a value of $33.33 \%$ (660 directed connections out of 1980) versus $24.36 \%$ in the younger adults. However, the above difference in the size or completeness of the CI network between the two groups was not significant ( $P=0.15$ for size and 0.21 for completeness, permutation testing). The network of the older adults was not in a small-world configuration $(S=0.85)$. Permutation testing showed that the small- 
world index of the older adults' network was significantly lower than that of the younger adults' network: the difference was highly significant for the left occipital module $(S=6.12)(P<0.001)$, and trend-level significant for the left fronto-temporal module $(S=1.88)$ in the younger adults' network ( $P$ $=0.05)$. These findings suggest that the brain circuitry in older adults may not be as efficient as younger adults to support retrieval of learned skills. The hubs of this network were the superior temporal gyrus (Temporal_Sup_L; BC = 0.26), the inferior frontal gyrus, opercular part (Frontal_Inf_Oper_L; BC = 0.14), and the insular cortex (Insula_L; BC $=0.13$ ) on the left, the paracentral lobule on the right (Paracentral_Lobule_R; $B C=0.12$ ), and the inferior frontal gyrus, triangular part, on the left (Frontal_Inf_Tri_L; BC $=0.10$ ). The CI network of the older adults had a modularity index $Q=0.21(Z$-score $=21.94)$, significantly lower than that $(Q=0.5)$ of the network of the younger adults ( $P<0.001$, permutation testing). Two modules were identified: module 1 covered parieto-occipital areas bilaterally, while module 2 was mostly in the left fronto-temporal regions.

(2) Higher network centrality was associated with stronger CI benefit to learning in younger but not older adults: For the younger adults, we found that when $3.8 \leq t_{c} \leq 4.5$, there was a significant positive correlation (with a $P$-value less than the Bonferroni's threshold) between the mean betweenness centrality (BC) of the participant-level CI networks on Day 5 and the adjusted R-I contrast in response time on the same day (Fig. 5a and Supplementary Table 3). Fig. 5b shows the most significant association (Spearman's rank correlation coefficient $\rho=0.75, P<0.001$ ), corresponding to $t_{c}=4.0$. By contrast, correlations for the adjusted R-I contrast in response time with the betweenness centrality of the CI network in the older adults were insignificant for all $t_{c}$ 's. The difference between the younger and older adults in the above correlations was significant, with a significant Group $\times$ CI interaction, when $4.0 \leq t_{c} \leq 4.5$ (Supplementary Table 3), indicating that better learning following interleaved 
practice was associated with higher nodal centrality in the younger but not older adults when weaker connections (connections with a $t$-value less than $t_{c}=4.0$ ) of the network were excluded.

\subsection{The CI networks based on seed regions related to the premotor (PMC) or dorsolateral} prefrontal cortices (DLPFC)

As a follow-up analysis of the global brain networks generated based on the AAL atlas above, we performed PPI based on seed regions functionally connected to the PMC or DLPFC during CI learning, and identified a 7-node CI network in the younger adults $(P=0.004$ corrected for multiple comparisons using the NBS method; Supplementary Figure 1). The network had only one hub, the temporal pole on the left $(\mathrm{BC}=0.27)$. This region is also one of the hubs in the AAL-based network for the younger adults as described above. Similar to the main findings with respect to the AAL-based networks, the younger adults' network was in a small-world topology, with the small-world index $S=2.19$. By contrast, the older adults' network was not considered to be a small-world network, as its small-world index $S=1.01$ was only at the borderline level. The older adults' network contained 23 nodes $(P<$ 0.0001 corrected for multiple comparisons), where the hubs of the network were the parietal operculum cortex $(\mathrm{BC}=0.35)$ and secondary somatosensory cortex $(\mathrm{BC}=0.11)$, both in the left hemisphere (Supplementary Figure 2).

\section{DISCUSSION}

In this paper we investigated how aging affects motor sequence learning, comparing differences in retention performance and brain networks between younger and older adults. The younger adults benefited more from interleaved training than older adults: they had a greater adjusted R-I contrast in retention RT, i.e., a shorter retention RT following interleaved practice when the retention RTs following repetitive practice in both participant groups were adjusted to the same level using ANCOVA. This CI benefit for motor learning in younger adults was associated with a more efficient brain network. Our main findings with respect to the seed regions from the AAL atlas showed three 
coherent characteristics in the CI network of the younger adults: (1) small-world topology that made communication across the network efficient; (2) stronger functional segregation, with a higher modularity index of the network; and (3) correlations between network centrality and the adjusted R-I contrast in the retention RT, indicating that better communication within the CI network was associated with a stronger CI benefit to motor learning.

\section{The CI network in younger but not older adults exhibited small-world topology}

In younger adults, introducing desirable difficulty into practice gave rise to an efficient motor learning network during retention - their CI network could be divided into two small-world subnetworks respectively in the left fronto-temporal and the left occipital lobes. Small-world topology has been associated with high efficiency in simultaneous information processing and low wiring costs (Rubinov and Sporns, 2010; Watts and Strogatz, 1998). Identification of small-world networks during retention in the younger adults suggests that the brain optimizes adaptive configuration of neural resources to support cognitive demands during motor learning with greater contextual interference (Jin et al., 2012). Moreover, a network being small-world means that most of the shortest paths in the network go through its hub nodes (Watts and Strogatz, 1998), i.e., the orbital part of the left inferior frontal gyrus and the left middle temporal pole for the younger adults. The orbital part of the left inferior frontal gyrus is traditionally a neural substrate of speech production. It may play an active role in CI learning through rehearsing or "verbalizing” the practiced sequences, a common strategy that has been identified in motor learning studies (Wright, 1991). The temporal pole is an important neural substrate for long-term memory, particularly the anterograde and retrograde declarative memory (Grabowski et al., 2001; Milner, 1954). Efficient functional connections between these two hub nodes therefore facilitate efficient retrieval of motor memory in the younger adults.

The CI network of the older adults did not exhibit the small-world configuration. The absence of a favorable small-world network in the older adults may be attributed to age-related decrease in synaptic function, which precludes efficient communication between remote neurons (Foster, 1999). 
Moreover, the hub nodes of the network involved sensorimotor areas: for example, the superior temporal gyrus integrates information registered through different sensory modalities (Kayser and Logothetis, 2009), while the paracentral lobule assists sensorimotor integration during programming of sequential finger movements (Iacoboni et al., 1998; Roland et al., 1980). This suggests that the older adults depended more on sensorimotor processing to accomplish the tasks during the retention phase, consistent with our previous observations (Lin et al., 2012a).

\section{The CI network in younger adults exhibited higher modularity than older adults}

In addition to the small-world property, the CI network in the younger adults was advantageous because it was highly modular. A modular brain network is economical in wiring costs, supporting fast adaptation to a new environment (Clune et al., 2013). Moreover, functional segregation of brain regions into different modules may reduce interference across different cognitive circuits, enabling the brain to respond to various stimuli more efficiently (Pessiglione et al., 2005). By contrast, the older adults had a lower modularity index and tended to recruit both hemispheres - their occipital module (module 1) covered bilateral parieto-occipital areas, while the occipital module (module 2) in the younger adults involved only the left hemisphere. This is consistent with previous neuroimaging findings that functional brain activation in older adults is usually more bilateral (Cabeza, 2002). Such bilateral recruitment may compensate for age-related decline in perceptual or visual processing in older adults (Cabeza, 2002).

\section{Better communication within the CI network was associated with a greater CI benefit on learning in younger adults}

We found that higher mean betweenness centrality (BC) in the CI network was associated with better retention performance following interleaved practice in the younger but not older adults. A network with a higher BC means that it has on average more nodes lying along the geodesic pathways of other nodes (Valente et al., 2008). As such, the positive correlation between the network centrality and retention performance implicates that CI training is a process of plastic remodeling in the brain 
network in younger adults, where learning is better when the information flow within the network is redirected more to those hub nodes. This is consistent with previous work showing that a higher level of global communication efficiency within functional brain networks was associated with a higher intellectual performance (van den Heuvel et al., 2009). It also suggests that increased complexity during motor practice such as CI training drives brain network reorganization. By contrast, the absence of such correlation in the older adults suggests that the efficiency of their brain network was not linked to retention performance, but as a result of complex interactions between age and CI training. The older adults may have lost flexibility to reconfigure its neural networks during the processes of memory consolidation. It is also possible that for older adults, motor learning may not induce a "fast flow" of information in the brain circuitry (i.e., most information within the network flows through the efficient hub nodes), which may account for their generally longer cognitive reaction times (Verhaeghen and Cerella, 2002). Alternatively, the CI training in the current design might not provide desirable difficulty to drive functional brain reconfiguration in the older adults.

\section{Limitations of the current study}

A couple of caveats should be noted with respect to the current findings. First, the fMRI data was smoothed using an 8-mm Gaussian kernel at the image preprocessing stage (see Supplementary Methods); this same size of the smoothing kernel has been used in previous PPI connectivity analyses, e.g., (Minati et al., 2012) and (Cocchi et al., 2014). Spatial smoothing has been commonly used in fMRI preprocessing to improve the signal-to-noise ratio and to compensate for imperfect normalization of the images (Lindquist, 2008). However, it may also enhance the similarity of time courses between adjacent voxels and therefore differentially influence specific network properties and bias the analysis towards detecting group differences. Nevertheless, such unfavorable effects of smoothing may be less prominent for low-resolution networks as studied in the current paper where the size of each node (a brain region defined on the AAL atlas) greatly exceeds the spatial range of the smoothing kernel (Zalesky et al., 2012). Second, aging is associated with gray matter atrophy and dilatation of the 
cerebrospinal fluid (CSF) space. Although it was found that atrophy in some brain regions, e.g., the language regions, did not affect fMRI activation in those regions in healthy older adults (Johnson et al., 2000), cortical atrophy in older adults in general may confound age-related BOLD differences due to distortion in spatial normalization and signal contamination from CSF (Samanez-Larkin and D'Esposito, 2008). The accuracy of spatial normalization may be improved by using population-specific templates for younger and older adults respectively (Buckner et al., 2004). However, the resulting coordinates in the population-specific templates may not match well to the standard Montreal Neurological Institute (MNI) space, so that the accuracy in delineation of regions of interest (ROI) on individual participants may be affected, particularly when the ROIs of individual participants are defined by warping the ROIs labeled on the standard MNI template (e.g., the AAL atlas used here) (Poldrack et al., 2011). Third,

older adults, for whatever reason, produce less robust or noisier hemodynamic responses (D'Esposito et al., 2003). For example, older adults tend to have more head motion than younger adults during MRI scanning (Van Dijk et al., 2012), which may not be fully corrected by nuisance regression of head motion parameters in the general linear model (GLM). As such, the observed differences in the network patterns between the younger and older adults might be biased by greater noise in the older adults’ data and should be carefully interpreted.

\section{Conclusion}

To our knowledge, this is the first study applying a graph theoretical analysis to demonstrate functional brain networks that underlie the beneficial effects of CI on learning. We have found that aging may interact with practice conditions to modulate functional brain networks essential for successful motor sequence learning. This agrees with the behavioral finding that practice with high CI did not benefit older adults as much as their younger counterpart. Our study may facilitate future designs of learning protocols to enhance the cognitive function of the aging brain. The network approaches used in this paper complement traditional methods, showing that learning not only 
modulates neural activity within individual regions, but also reorganizes the whole functional circuits across the brain.

Acknowledgments. The authors declare no competing financial interests. This study was supported in part by the Ministry of Science and Technology (MOST 102-2221-E-010-007-MY3), the National Health Research Institutes (NHRI-EX104-10219EC), and the Brain Research Center, National YangMing University (a grant from Ministry of Education, Aim for the Top University Plan, 100AC-B12), Taiwan (to MC Chiang), American Heart Association and Center of Experimental Neurorehabilitation Training (CJ Lin, AD Wu), National Science Foundation (BJ Knowlton) and Howard Hughes Medical Institutes (AD Wu).

\section{REFERENCES}

Bassett DS, Bullmore E. Small-world brain networks. Neuroscientist 2006; 12(6): 512-23.

Buckner RL, Head D, Parker J, Fotenos AF, Marcus D, Morris JC, et al. A unified approach for morphometric and functional data analysis in young, old, and demented adults using automated atlas-based head size normalization: reliability and validation against manual measurement of total intracranial volume. Neuroimage 2004; 23(2): 724-38.

Cabeza R. Hemispheric asymmetry reduction in older adults: the HAROLD model. Psychol Aging 2002; 17(1): 85-100.

Christina RW, Bjork RA. Optimizing long-term retention and transfer. In: Druckman D, Bjork RA, editors. In the mind's eye: Enhancing human performance Washington DC: National Academy Press; 1991. p. 23-56.

Clune J, Mouret JB, Lipson H. The evolutionary origins of modularity. Proc Biol Sci 2013; 280(1755): 20122863. 
Cocchi L, Halford GS, Zalesky A, Harding IH, Ramm BJ, Cutmore T, et al. Complexity in Relational Processing Predicts Changes in Functional Brain Network Dynamics. Cereb Cortex 2014; 24(9): 2283-96.

Cross ES, Schmitt PJ, Grafton ST. Neural substrates of contextual interference during motor learning support a model of active preparation. J Cogn Neurosci 2007; 19(11): 1854-71.

D'Esposito M, Deouell LY, Gazzaley A. Alterations in the BOLD fMRI signal with ageing and disease: a challenge for neuroimaging. Nat Rev Neurosci 2003; 4(11): 863-72.

Folstein MF, Folstein SE, McHugh PR. "Mini-mental state". A practical method for grading the cognitive state of patients for the clinician. J Psychiatr Res 1975; 12(3): 189-98.

Foster TC. Involvement of hippocampal synaptic plasticity in age-related memory decline. Brain Res Brain Res Rev 1999; 30(3): 236-49.

Friston KJ, Buechel C, Fink GR, Morris J, Rolls E, Dolan RJ. Psychophysiological and modulatory interactions in neuroimaging. Neuroimage 1997; 6(3): 218-29.

Gerchen MF, Bernal-Casas D, Kirsch P. Analyzing task-dependent brain network changes by wholebrain psychophysiological interactions: a comparison to conventional analysis. Hum Brain Mapp 2014; 35(10): 5071-82.

Gitelman DR, Penny WD, Ashburner J, Friston KJ. Modeling regional and psychophysiologic interactions in fMRI: the importance of hemodynamic deconvolution. Neuroimage 2003; 19(1): 200-7.

Grabowski TJ, Damasio H, Tranel D, Ponto LL, Hichwa RD, Damasio AR. A role for left temporal pole in the retrieval of words for unique entities. Hum Brain Mapp 2001; 13(4): 199-212.

He Y, Wang J, Wang L, Chen ZJ, Yan C, Yang H, et al. Uncovering intrinsic modular organization of spontaneous brain activity in humans. PLoS One 2009; 4(4): e5226.

Humphries MD, Gurney K. Network 'small-world-ness': a quantitative method for determining canonical network equivalence. PLoS One 2008; 3(4): e0002051. 
Iacoboni M, Woods RP, Mazziotta JC. Bimodal (auditory and visual) left frontoparietal circuitry for sensorimotor integration and sensorimotor learning. Brain 1998; 121 ( Pt 11): 2135-43.

Jin SH, Lin P, Hallett M. Reorganization of brain functional small-world networks during finger movements. Hum Brain Mapp 2012; 33(4): 861-72.

Johnson SC, Saykin AJ, Baxter LC, Flashman LA, Santulli RB, McAllister TW, et al. The relationship between fMRI activation and cerebral atrophy: comparison of normal aging and alzheimer disease. Neuroimage 2000; 11(3): 179-87.

Kayser C, Logothetis NK. Directed Interactions Between Auditory and Superior Temporal Cortices and their Role in Sensory Integration. Front Integr Neurosci 2009; 3: 7.

Langer N, Pedroni A, Jancke L. The problem of thresholding in small-world network analysis. PLoS One 2013; 8(1): e53199.

Lin $\mathrm{CH}$, Chiang MC, Knowlton BJ, Iacoboni M, Udompholkul P, Wu AD. Interleaved practice enhances skill learning and the functional connectivity of fronto-parietal networks. Hum Brain Mapp 2013; 34(7): 1542-58.

Lin CH, Chiang MC, Wu AD, Iacoboni M, Udompholkul P, Yazdanshenas O, et al. Age related differences in the neural substrates of motor sequence learning after interleaved and repetitive practice. Neuroimage 2012a; 62(3): 2007-20.

Lin $\mathrm{CH}$, Chiang MC, Wu AD, Iacoboni M, Udompholkul P, Yazdanshenas O, et al. Enhanced motor learning in older adults is accompanied by increased bilateral frontal and fronto-parietal connectivity. Brain Connect 2012b; 2(2): 56-68.

Lin $\mathrm{CH}, \mathrm{Wu} \mathrm{AD}$, Udompholkul P, Knowlton BJ. Contextual interference effects in sequence learning for young and older adults. Psychol Aging 2010; 25(4): 929-39.

Lindquist MA. The statistical analysis of fMRI data. Stat Sci 2008; 23(4): 439-64.

Milner B. Intellectual function of the temporal lobes. Psychol Bull 1954; 51(1): 42-62. 
Minati L, Grisoli M, Seth AK, Critchley HD. Decision-making under risk: a graph-based network analysis using functional MRI. Neuroimage 2012; 60(4): 2191-205.

Nieuwenhuis S, Forstmann BU, Wagenmakers EJ. Erroneous analyses of interactions in neuroscience: a problem of significance. Nat Neurosci 2011; 14(9): 1105-7.

Pessiglione M, Guehl D, Rolland AS, Francois C, Hirsch EC, Feger J, et al. Thalamic neuronal activity in dopamine-depleted primates: evidence for a loss of functional segregation within basal ganglia circuits. J Neurosci 2005; 25(6): 1523-31.

Poldrack RA, Mumford JA, Nichols TE. Handbook of functional MRI data analysis. New York: Cambridge University Press; 2011.

Roland PE, Larsen B, Lassen NA, Skinhoj E. Supplementary motor area and other cortical areas in organization of voluntary movements in man. J Neurophysiol 1980; 43(1): 118-36.

Rubinov M, Sporns O. Complex network measures of brain connectivity: uses and interpretations. Neuroimage 2010; 52(3): 1059-69.

Samanez-Larkin GR, D'Esposito M. Group comparisons: imaging the aging brain. Soc Cogn Affect Neurosci 2008; 3(3): 290-7.

Sami S, Miall RC. Graph network analysis of immediate motor-learning induced changes in resting state BOLD. Front Hum Neurosci 2013; 7: 166.

Shea JB, Morgan RL. Contextual interference effects on the acquisition, retention, and transfer of a motor skill. J Exp Psychol Hum Learn 1979; 5(2): 179-87.

Stanley ML, Moussa MN, Paolini BM, Lyday RG, Burdette JH, Laurienti PJ. Defining nodes in complex brain networks. Front Comput Neurosci 2013; 7: 169.

Supekar K, Menon V, Rubin D, Musen M, Greicius MD. Network analysis of intrinsic functional brain connectivity in Alzheimer's disease. PLoS Comput Biol 2008; 4(6): e1000100. 
Testa A, Furtado M, Alipour A. 2014. Seismic resilience assessment of highway networks: a topologybased approach. 2nd European Conference on Earthquake Engineering and Seismology, Istanbul, Turkey.

Valente TW, Coronges K, Lakon C, Costenbader E. How Correlated Are Network Centrality Measures? Connect (Tor) 2008; 28(1): 16-26.

van den Heuvel MP, Stam CJ, Kahn RS, Hulshoff Pol HE. Efficiency of functional brain networks and intellectual performance. J Neurosci 2009; 29(23): 7619-24.

Van Dijk KR, Sabuncu MR, Buckner RL. The influence of head motion on intrinsic functional connectivity MRI. Neuroimage 2012; 59(1): 431-8.

Verhaeghen P, Cerella J. Aging, executive control, and attention: a review of meta-analyses. Neurosci Biobehav Rev 2002; 26(7): 849-57.

Vickers AJ. The use of percentage change from baseline as an outcome in a controlled trial is statistically inefficient: a simulation study. BMC Med Res Methodol 2001; 1(1): 6.

Watts DJ, Strogatz SH. Collective dynamics of 'small-world' networks. Nature 1998; 393(6684): 440-2. Wright DL. The role of intertask and intratask processing in acquisition and retention of motor skills. J Mot Behav 1991; 23(2): 139-45.

Yan C, He Y. Driving and driven architectures of directed small-world human brain functional networks. PLoS One 2011; 6(8): e23460.

Zalesky A, Fornito A, Bullmore E. On the use of correlation as a measure of network connectivity. Neuroimage 2012; 60(4): 2096-106.

Zalesky A, Fornito A, Bullmore ET. Network-based statistic: identifying differences in brain networks. Neuroimage 2010; 53(4): 1197-207. 


\section{FIGURE LEGENDS}

Figure 1. Experimental design and functional network analysis. (a) During fMRI scanning, the participants practiced the SRT task by pressing the corresponding key with their left hand as soon as they saw the colored circles appearing one by one through the magnet-compatible goggles. (b) The SRT task included two sets of three different four-element sequences, $(1,2,3)$ and $(4,5,6)$. Each element of a sequence was represented by a circle colored in blue (B), red (R), green (G), and yellow (Y). (c) Each participant was assigned to either the Repetitive or the Interleaved training group during the 5-day regime of the SRT task (practice of the task on Days 1 and 2; retention tests on Day 5). In the Repetitive condition, a sequence was practiced repetitively for 54 consecutive trials before the next sequence appeared (e.g., sequences $111 \ldots 222 \ldots 333 \ldots$ ), while in the Interleaved condition, the three sequences (sequences 4, 5, and 6) were arranged in a non-repetitive order. During the retention tests on Day 5, the practiced sequences were presented in a repetitive order in the first fMRI run (shown by $1 \ldots 2 \ldots 3 \ldots$ for the Repetitive, and $4 \ldots 5 \ldots 6 \ldots$ for the Interleaved training group), but in an interleaved order in the second fMRI run (shown by 132...for the Repetitive, and 465... for the Interleaved training group). The participants were tested with three novel sequences (denoted by 'Novel') in the third fMRI run. However, data from this run was not used in this paper. (d) Psychophysiological Interaction (PPI) analysis was applied to fMRI data acquired on Day 5 to estimate functional connectivity between any pair of brain regions $[(\mathrm{d})$, left]. If functional connectivity from the seed to the target region was greater in the Interleaving training, the regression slope for the target against the seed fMRI activity would be steeper in the Interleaved (black) than the Repetitive (red) training condition [(d), right]. (e) Functional connectivity of the brain was visualized as a connectivity matrix color-coded by $t$-values that gauged the significance of excess in functional connectivity for the Interleaved over the Repetitive condition from the seed (row) to the target (column) region. (f) Finally, the connectivity matrix in (e) was converted to a directed network mediating the CI effect on learning, i.e., the CI network. 
Figure 2. Associations between CI effects on motor learning and small-world topology of brain networks. (a) Both younger and older adults exhibited CI effects on motor learning, as shown by a shorter mean response time (RT) following interleaved practice (left panel). However, the difference between the younger and older adults in the values of the Repetitive minus the Interleaved RT ('R-I contrast') was not significant (right panel). (b) When retention RT following interleaved practice was compared on the same level of RT following repetitive practice (i.e., $\overline{\mathbf{t}}_{\mathbf{R P}}$ ) under the ANCOVA model (left panel), the younger adults showed a significantly greater adjusted R-I contrast, indicating that they benefited more than older adults from the practice with higher contextual interference (right panel). Error bars in (a) and (b) indicate mean \pm standard error. (c) The greater CI benefit to learning in the younger adults was associated with a small-world topology in their brain network, visualized using the BrainNet Viewer (http://www.nitrc.org/projects/bnv/). The CI network of the younger adults was divided into two separate modules, the left fronto-temporal module (colored in green) and the left occipital module (colored in red). Both modules were in a small-world topology: the small-world index $S_{1}=1.88$ in the left fronto-temporal module, and $S_{2}=6.12$ in the left occipital module. The CI network of the older adults contained two modules: module 1 covered parieto-occipital areas bilaterally (green), while module 2 was mostly in the left fronto-temporal regions (red). In contrast to the younger adults, the older adults did not have a small-world network $(S=0.85)$. Arrows define the directed connection from the seed to the target region. The hub nodes were displayed in a larger size with their abbreviated name labeled. Their full name was listed in Supplementary Table 1. ' $\mathrm{R}$ ' and 'L': right and left hemisphere.

Figure 3. The connectivity matrix of the younger adults on Day 5. The connectivity matrix for the younger adults, as well as that for the older adults in Fig. 4, was derived from the group-level PPI analysis across all the $88 \mathrm{AAL}$ atlas regions, yielding a $t$-value (color-coded) that quantified the significance of excess in functional connectivity for the Interleaved minus Repetitive condition, for any 
pair of the seed (row) and the target (column) regions. The connectivity matrix was further corrected for multiple comparisons using the NBS toolbox, giving a final size of the matrix equal to $13 \times 13$. The nodes of the network were colored according to the module they belonged to; those in module 1 (left fronto-temporal module) were colored in green, and module 2 (left occipital module) in red. The hub nodes were labeled with solid circles. The full name of the nodes was listed in Supplementary Table 1. Figure 4. The connectivity matrix of the older adults on Day 5. The definitions and settings of the connectivity matrix are the same as in Fig. 3. The full name of the nodes was listed in Supplementary

\section{Table 1.}

\section{Figure 5. Associations between brain network centrality and CI effect on learning in the younger}

adults. (a) The line chart shows the Spearman's rank correlation coefficient $\rho$ between the younger participants' adjusted R-I contrast on Day 5 and the mean betweenness centrality (BC) of the CI network on the same day when the participant-level $t$-contrast matrices derived from PPI were thresholded at $t_{c}$ ranging from 3.0 to 4.5 . The asterisks $\left(^{*}\right)$ indicate that the correlation coefficient $\rho$ is significant at $3.8 \leq t_{c} \leq 4.5$, while the pound signs (\#) indicate significant Group $\times$ CI interactions at 4.0 $\leq t_{c} \leq 4.5$, i.e., the differences between the younger and older adults in the above associations between the learning benefit and network centrality are significant. All these significance levels have been corrected for the $16 t_{c}$ 's using the Bonferroni's method. (b) The correlation is most significant when $t_{c}=$ $4.0(\rho=0.75 ; P<0.001)$, as demonstrated in the scatter plot. These findings indicate that participants with higher centrality, or better communication in their CI network were more likely to show better retention performance following interleaved practice. 
Figure 1

(a) Serial reaction time task

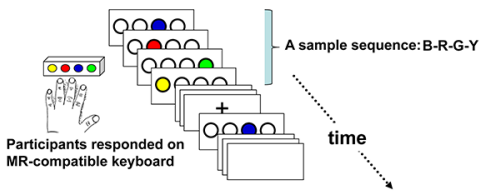

(b) Sample sequences

1 Y-G-B-R 4 G-B-Y-R

2 B-R-G-Y 5 Y-R-G-B

3 R-G-B-Y 6 B-G-R-Y

(c) 5-day regime of practice

Repetitive training

\begin{tabular}{|c|c|c|c|c|c|c|}
\hline fMRI & Run1 & Run2 & Run3 & Run1 & Run2 & Run3 \\
\hline Tas & $111 \ldots$ & $222 \ldots$ & 333... & $111 \ldots$ & $222 \ldots$ & $333 \ldots$ \\
\hline & $\begin{array}{l}54 \text { trials } \\
\text { Interle }\end{array}$ & $\begin{array}{l}54 \text { trials } \\
\text { aved tra }\end{array}$ & $\begin{array}{l}54 \text { trials } \\
\text { ining }\end{array}$ & 54 trials & 54 trials & 54 trials \\
\hline & Run1 & Run2 & Run3 & Run1 & Run2 & Run3 \\
\hline$T$ & $456 \ldots$ & $465 \ldots$ & $546 \ldots$ & $456 \ldots$ & $465 \ldots$ & 546. \\
\hline
\end{tabular}

$\mathbf{5 4}$ trials $\mathbf{5 4}$ trials $\mathbf{5 4}$ trials $\mathbf{5 4}$ trials $\mathbf{5 4}$ trials $\mathbf{5 4}$ trials

\section{(d) PPI analysis}
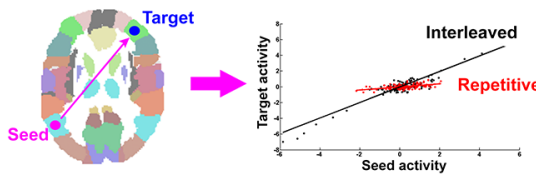

(e) Connectivity matrix

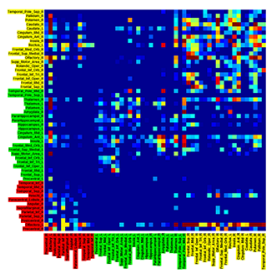

\begin{tabular}{l|l|l|} 
Run1 & Run2 & Run3 \\
\hline 1.2 .3$. & $132 .$. & Tovel \\
\hline
\end{tabular}

\begin{tabular}{|l|l|l|}
\hline 1..2.3. & $132 \ldots$ & Novel \\
\hline
\end{tabular}

36 trials 36 trials 36 trials

\begin{tabular}{l|l|l|}
\hline Run1 Run2 & Run3 \\
\hline
\end{tabular}

\begin{tabular}{|l|l|l|}
\hline $4.5 . .6$ & $465 \ldots$ & Novel \\
\hline
\end{tabular}

36 trials 36 trials 36 trials

Day 5 (f) Brain networks
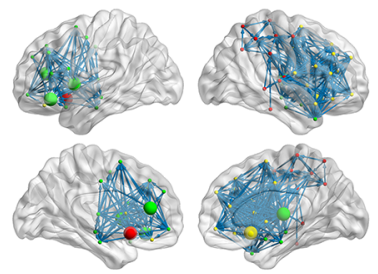


\section{Figure 2}

a

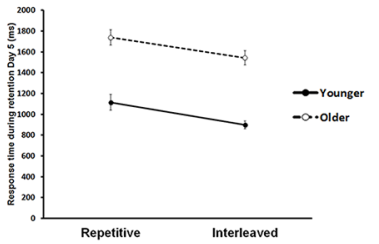

R-I contrast

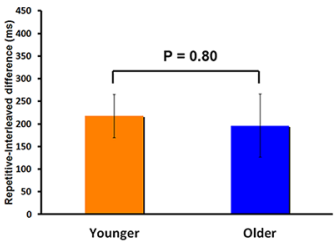

b

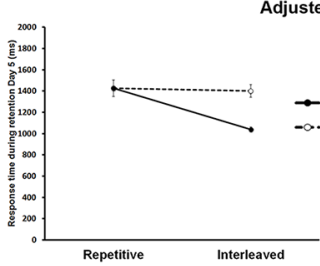

C
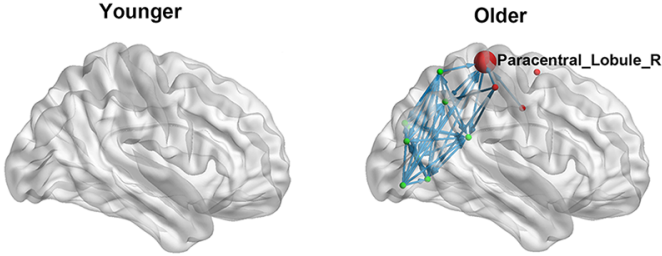

$\mathbf{R}$

Frontal_unf 0
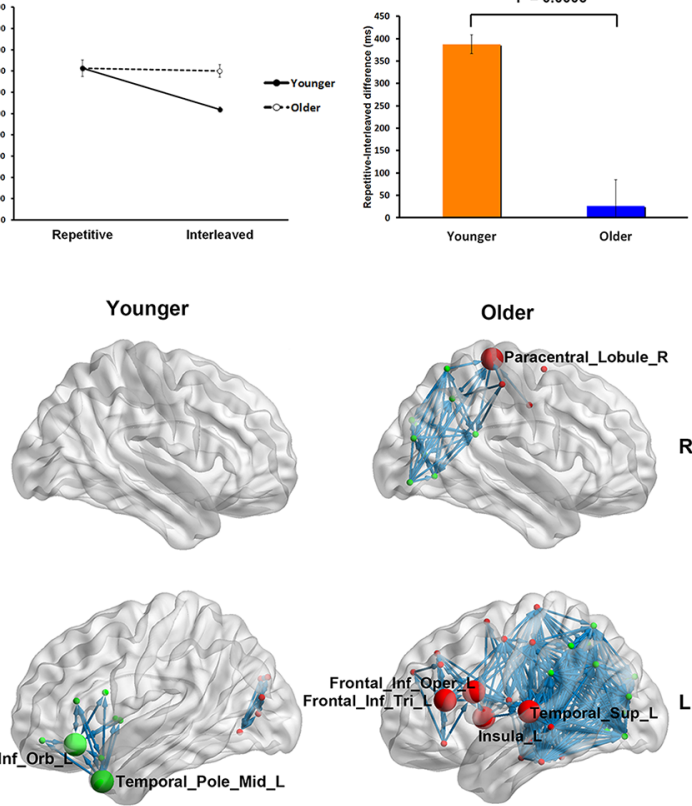

$$
\begin{aligned}
& S 1=1.88 \\
& S 2=6.12
\end{aligned}
$$


Figure 3

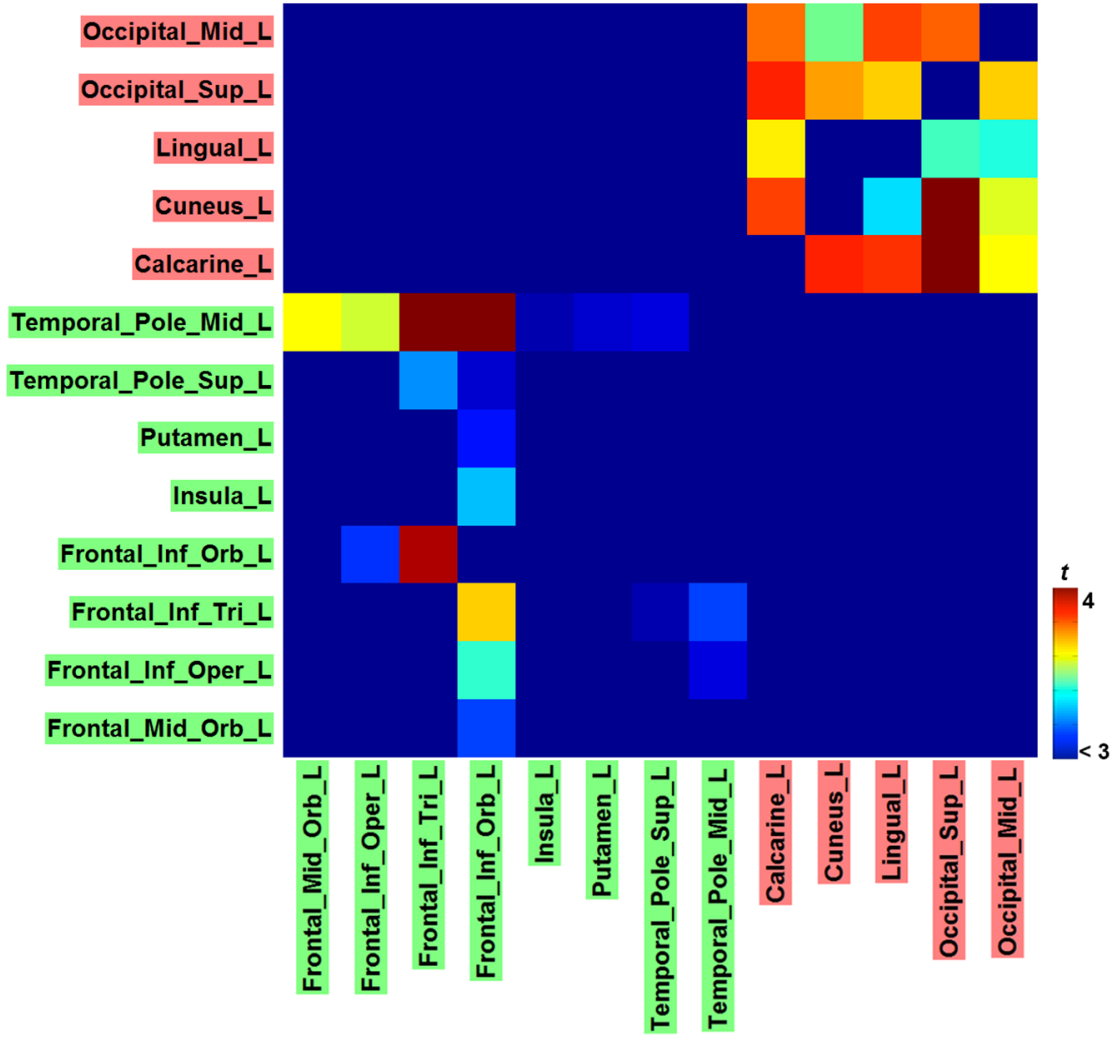




\section{Figure 4}

\begin{tabular}{l|l|l|l|l}
\hline & Module 1 & Module 2
\end{tabular}

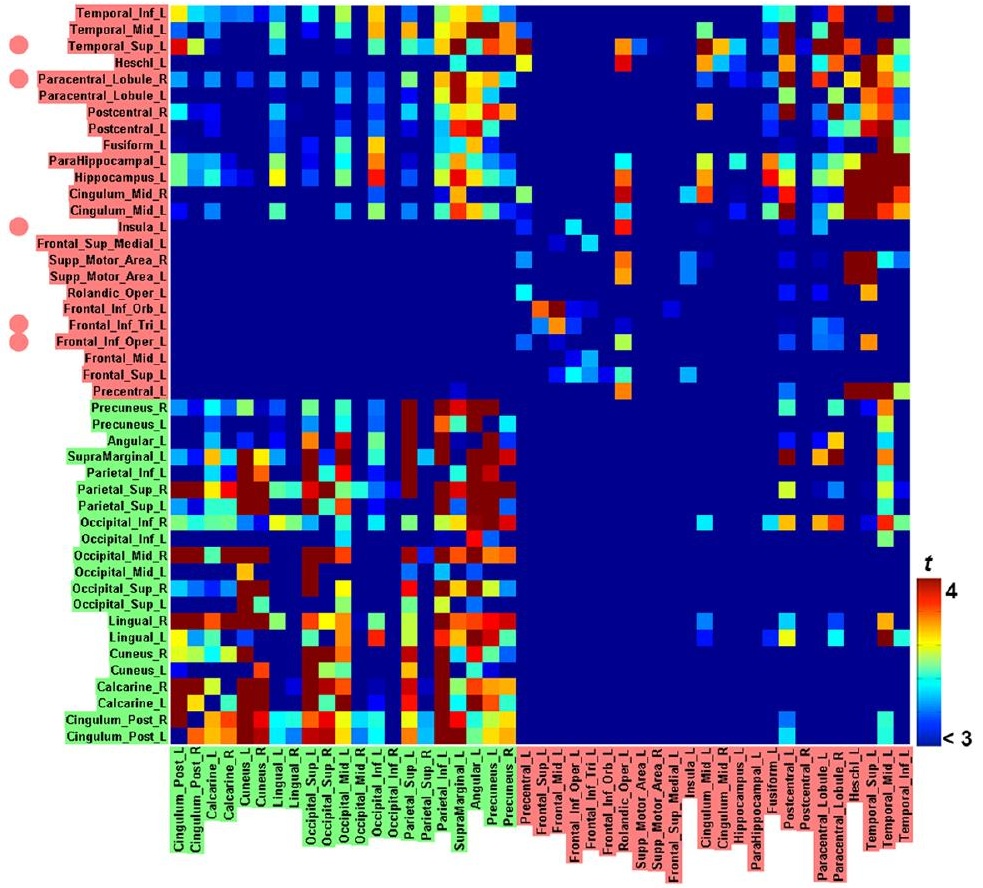




\section{Figure 5}

a

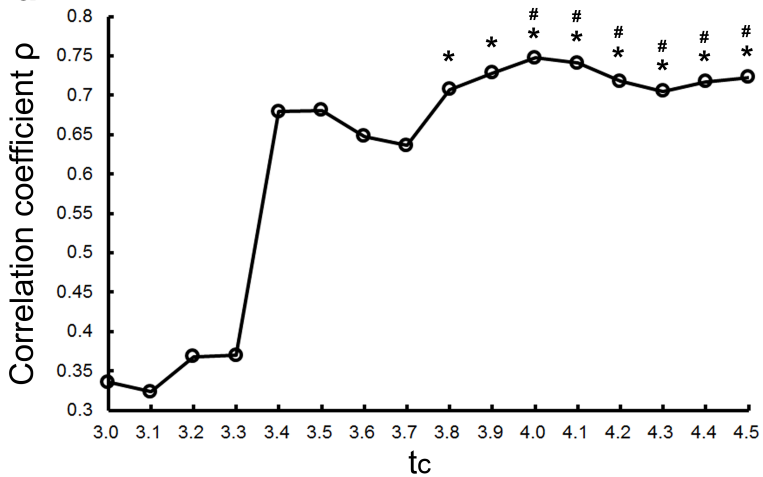

b

tc $=4.0$

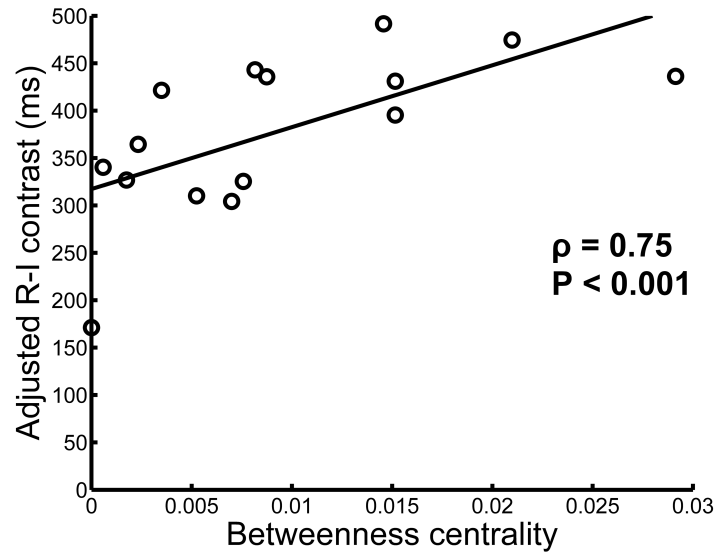

